\title{
Erratum
}

\section{A Modeling Study of Notch Noise Responses of Type III Units in the Gerbil Dorsal Cochlear Nucleus}

\author{
XiaOhan Zheng and Herbert F. Voigt \\ Biomedical Engineering Department and Hearing Research Center, Boston University, \\ 44 Cummington Street, Boston, MA 02215 \\ (Published online: 11 October 2006)
}

\begin{abstract}
A computational model of the neural circuitry of the gerbil dorsal cochlear nucleus (DCN), based on the MacGregor's neuromime model, was used to simulate type III unit (P-cell) responses to notch noise stimuli. The DCN patch model is based on a previous computational model of the cat DCN [Hancock, K. E., and H. F. Voigt. Ann. Biomed. Eng. 27:73-87, 1999]. According to the experimental study of Parsons et al. [Ann. Biomed. Eng. 29:887-896, 2001], the responses of gerbil DCN type III units to notch noise stimuli are similar to those of cat DCN type IV units, which are thought to be spectral notch detectors. This suggests that type III units in the gerbil DCN may serve as spectral notch detectors. In this modeling study, a simplified notch noise response plot-spike discharge rate vs. notch cutoff frequency plot-was used to compare model responses to the experimental results. Parameter estimation and sensitivity analysis of three connection parameters within the DCN patch have been studied and shows the model is robust, providing reasonable fits to the experimental data from 14 of 15 type III units examined [work supported by a grant from NIDCD, Boston University's Biomedical Engineering department and Hearing Research Center].
\end{abstract}

Keywords-Hearing, Sound localization, Parameter estimation, Sensitivity analysis.

\section{INTRODUCTION}

The cochlear nucleus $(\mathrm{CN})$ is the sole target of auditory nerve fibers and is the first brainstem nucleus in the ascending auditory pathway. The dorsal part of the cochlear nucleus (DCN) is one of its three sub-nuclei and has initial integration functions. The DCN's role in audition appears, in part, to be involved in sound localization in the median plane equidistant from the two ears where the traditional sound source location cues, interaural time and level differences, are absent. For broadband signals, the pinna attenuates energy in a narrow band whose center frequency can be correlated with sound source elevation.

\footnotetext{
*Originaly published Online First February 9, 2006, DOI: 10.1007/s10439-005-9073-5. This article was originally published online in an uncorrected form. The corrected article is reprinted in its entirety here.

Address correspondence to Herbert F. Voigt, Ph.D., Department of Biomedical Engineering, Boston University, 44 Cummington Street, Boston, MA 02215-2407 Electronic mail: hfv@bu.edu
}

The pinnae-induced attenuation is called a spectral notch. These notches are seen in the head-related transfer functions (HRTFs), which are the Fourier transforms of the impulse responses from free space sound sources to the tympanic membrane. These spectral features are found in human, cat and gerbil HRTFs. ${ }^{12,13}$ Principal cells of the DCN, type III units in the gerbil ${ }^{15}$ and type IV units in the cat, ${ }^{22}$ exhibit sharp sensitivity to notch noise signals. ${ }^{14,18}$ This suggests that the DCN is a spectral notch detector across different species.

The previous study of Hancock and Voigt ${ }^{6}$ used a computational model of the DCN to model the responses of three type IV units to notch noises and presented close fits to the physiological data recorded by Spirou and Young. ${ }^{18}$ The gerbil DCN has fewer type IV units than the cat DCN $(11 \%$ type IV units in gerbil vs. $31 \%$ in cat $)^{2,17}$ and is comprised primarily of type III units $(62 \%){ }^{2}$ As the principal units in gerbil DCN, type III units undoubtedly play an important role in gerbil sound localization. In this study, we used the same computational model to fit the physiological data obtained from the gerbil DCN by Parsons et al. ${ }^{15}$ Despite the fact that type III units have quite different responses to tones than type IV units, this study shows that by modifying the connectivities within the neuron groups we can simulate both type III and type IV units as notch detectors. Also in this work we have good fits for 14 out of 15 type III units in gerbil; this is substantially more modeling data than previously presented and points to the robustness of the DCN model.

\section{RESPONSE MAP SCHEME AND RATE VS. CUTOFF FREQUENCY PLOTS}

A response map (RM) is a plot of excitatory and inhibitory responses to tonal stimuli shown in a stimulus frequency vs. sound pressure level plane. It is a popular method to categorize the neurons in the $\mathrm{CN}$ of unanethetized, decerebrate animals. ${ }^{4,17,22,23}$ Type III units are characterized by their RMs (Fig. 1A), which show a center V-shape excitation region flanked by side-band inhibition. The tip of the excitation region points to the unit's best frequency 


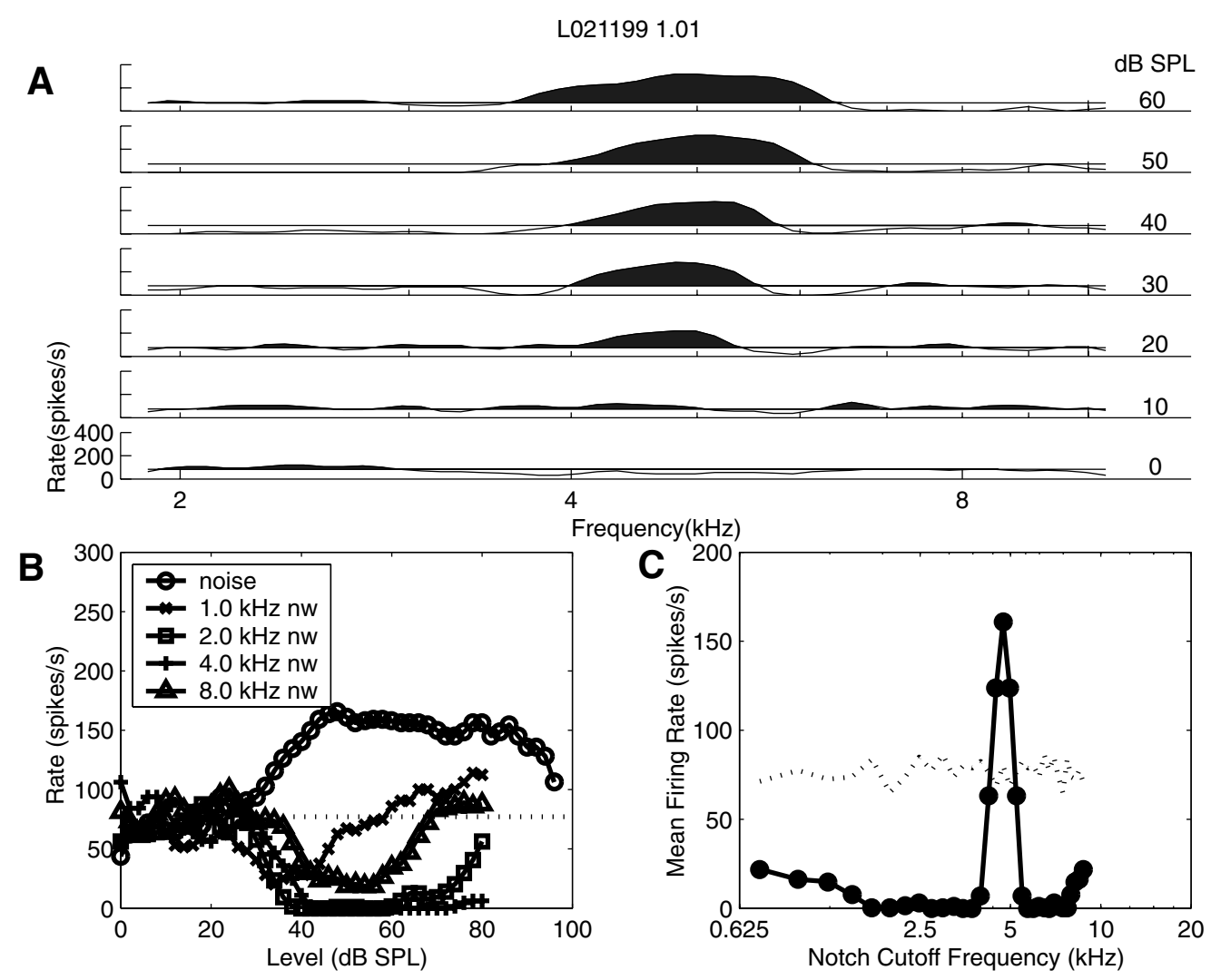

FIGURE 1. Physiology of a type III unit: (A) Response map composed of rate vs. frequency curves of unit L021199 1.01 in Parsons et al. ${ }^{15}$. Excitatory responses are shaded black while inhibitory responses left blank. (B) Rate-level curves when given wideband noise and notch noise stimuli. Only four notch noise responses with notch (nw) widths $1.0 \mathrm{kHz}, 2.0 \mathrm{kHz}, 4.0 \mathrm{kHz}$, and $8.0 \mathrm{kHz}$ are shown. (C) Rate vs. Cutoff frequency plot. Here notch widths varies systematically from 0.5 to $8.0 \mathrm{kHz}$ in step of $0.5 \mathrm{kHz}$. The average rates are calculated from the range of $46-54 \mathrm{~dB}$ SPL as shown by black bar in (B).

(BF), the frequency to which the unit was most sensitive. In Fig. 1A, there are seven discharge-rate vs. frequency curves shown for seven sound pressure levels presented. RMs of type IV units typically show an excitatory region over BF and an inhibitory area above that ranging over a broad frequency range; sometimes a narrow excitatory area appears off BF (see Fig. 1 of Ref. ${ }^{6}$ ).

Responses to broadband noise, in addition to tones, are used to classify units in the DCN. We also used the same notch noise (NN) stimuli in the model simulations as in the physiological experiments. ${ }^{15}$ These have band reject notches of $30 \mathrm{~dB}$ centered at the BF with varying widths. In Fig. 1B, discharge-rate vs. level curves are plotted for broadband noise and four NN stimuli with widths of 1,2 , 4 , and $8 \mathrm{kHz}$. These responses were taken from a series of responses to BF-centered NN stimuli whose widths systematically varied from 0.5 to $8.5 \mathrm{kHz}$ with a $0.5 \mathrm{kHz}$ step. For Unit L021199 1.01 in Fig. 1 there were $16 \mathrm{NN}$ stimuli applied with widths from 0.5 to $8.0 \mathrm{kHz}$. Only four NN responses are plotted for clarity.

To summarize the information in these rate-level curves, Spirou and Young ${ }^{18}$ further characterized NN responses with rate vs. cutoff frequency plots (Fig. 1C), where the firing rates to each $\mathrm{NN}$ were averaged over a range of sound levels from 46 to $54 \mathrm{~dB}$ SPL, where the inhibition reached the maximum, as indicated with black bar on the abscissa in Fig. 1B. The cutoff frequencies included both the notch noise's lowpass and the highpass cutoff frequencies, thus the average rate of each notch noise response is plotted twice. In such plots, 11 of 15 units in Parsons et al.'s experimental data showed a specific "Mexican Hat" shape, indicating that when increasing the notch width, the average response rate dropped sharply from excitatory to inhibitory and then showed some degree of recovery. The unit in the figure was excited by the broad-band noise and $\mathrm{NN}$ with narrow-notch width $(0.5 \mathrm{kHz}$, not shown in Fig. 1B but shown in Fig. 1C), but was inhibited by $\mathrm{NN}$ with wider widths (from 1.0 to $6.0 \mathrm{kHz}$, also shown in Fig. 1C). Also it showed some degree of recovery to a less inhibitory stage when NN with an even wider notch was applied (from 6.5 to $8.0 \mathrm{kHz}$, shown in Fig. 1C). This recovery was seen in 11 of 15 units in the paper of Parsons et al. ${ }^{15}$ Similar responses to NN have been found in type IV units in cat by Spirou and Young. ${ }^{18}$ 


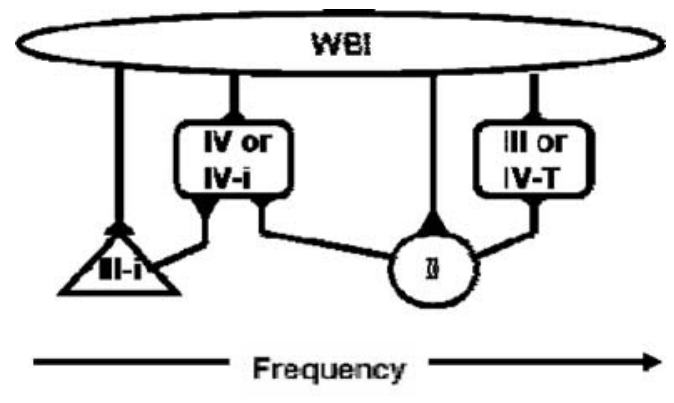

FIGURE 2. Conceptual model of gerbil dorsal cochlear nucleus circuitry. Only inhibitory connections are shown. The size of the triangles indicates connection strength. The horizontal axis represents the input from the auditory nerve fibers with increasing frequency, which target all cells. Modified from Davis et al. ${ }^{2}$

This demonstrated the sensitivity of type III units in gerbils to the NNs. Thus, type III units in gerbils are capable of detecting spectral notches as do type IV units in cats.

\section{CONCEPTUAL MODEL OF DCN CIRCUITRY}

To illustrate the functional role of type III units in the gerbil DCN, the conceptual model from Davis et al. ${ }^{2}$ was chosen (see Fig. 2). The gerbil DCN conceptual model is more complex than the cat model since the principal cells in gerbils can be either type III units or type IV units, including subtypes of type IV units: type IV-i and type IV$T$ units. In addition, besides type II units and the wide band inhibitor (WBI) units, type III-i units were included in the circuitry to provide necessary inhibitory input to type IV-i units. In this paper, however, we only discuss the properties and responses of type III units, thus only type II units and WBI units are taken as inhibitors.

Auditory nerve (AN) fibers provide excitatory inputs to type II units, type III units and WBI units. However, DCN units show spontaneous activity even after destruction of the cochlea. ${ }^{9}$ Thus non-specific afferents (NSA) were added to the principal cells in our computational model to provide noncochlear inputs to the DCN (Fig. 3). In order not to confuse physiologically defined unit types and model unit types, we use different names to distinguish them (see Table 1).

Fusiform cells in gerbil DCN are mostly type III units in intracellular recording and marking experiments by Hancock and Voigt ${ }^{7}$ whereas type II units arise from vertical cells in the $\mathrm{DCN}^{21}$ and WBI units are thought to project from the posteroventral $\mathrm{CN} .{ }^{20}$ In addition, WBI units send inhibitory connections to both type IV units (principal units in cat) and type II units in cat DCN. We are assuming that these connections to the gerbil principal units remain intact.

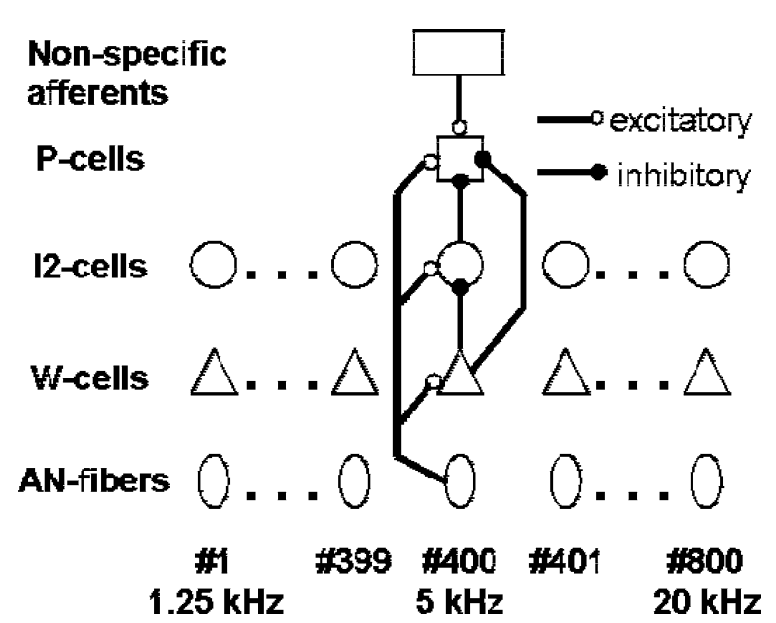

FIGURE 3. A patch of DCN model. Five cell populations are organized in a tonotopic manner with frequency step equal to 0.005 octaves. The excitatory and inhibitory connections are shown in a single frequency slice. $P$ cells, representing type III units in gerbil and type IV units in cat; 12 cells, type II units; W cells, providing wide band inhibition; AN fibers, the auditory nerve fiber input. Modified From Hancock and Voigt, 1999. ${ }^{6}$

\section{METHODS}

\section{Organization of the DCN Model}

The gerbil model is based on a previous computational model of the cat $\mathrm{DCN}^{6}$ (see Fig. 3). The model contains five cell groups that are arranged into 800 isofrequency slices centered at $5 \mathrm{kHz}$ with two octaves below and above in intervals of 0.005 octaves. Figure 3 shows the connections among the model cells within a single frequency slice, where P-cells represent the principal cells, I2-cells represent interneuron inhibitors with type II responses and W-cells represent wide band inhibitors. AN-fibers excite $\mathrm{P}$-cells, I2-cells and W-cells. W-cells inhibit P-cells and I2-cells. I2-cells inhibit P-cells. P-cells also are excited by non-specific afferents.

The relationship among the model cells can be discussed in terms of the connection parameters as shown in Fig. 4. For a given population of model cells (e.g., P-cells), the target cell "B" will receive $\mathrm{N}$ inputs from the "A" source cells, which are chosen from a band of "A" source cells with bandwidth BW, offset from the BF of B by C. The

TABLE 1. Physiological unit types with corresponding model unit types.

\begin{tabular}{ll}
\hline Physiological unit type & Model unit type \\
\hline Type III units & P-cells \\
Type II units & I2-cells \\
Wideband inhibitors & W-cells \\
Auditory nerve fibers & AN-fibers
\end{tabular}




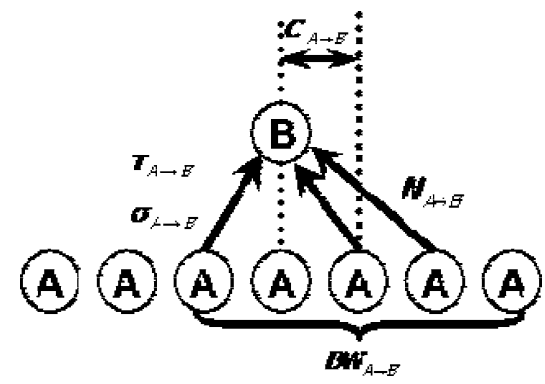

FIGURE 4. Group connection parameters. $B$ is the target cell and a group of source cells $A$ connect to it. $\sigma_{A \rightarrow B}$ and $\tau_{A \rightarrow B}$ are the step increase and time constant in response to the input spikes. Here $B$ cell receives $\mathrm{N}$ inputs from $\mathrm{A}$ cells, which are randomly chosen from a band of bandwidth and center frequency offset specified cells.

subscript $\mathrm{A} \rightarrow \mathrm{B}$ indicates the source unit, A to the target unit, B. In the simulations shown here, all the P-cells' BFs will be $5 \mathrm{kHz}$. ]

\section{Neuron Model}

The neuron model (Fig. 5) is based on the MacGregor neuromime model,${ }^{10}$ which is a parallel circuit model containing the membrane capacitance, leakage conductance, a potassium channel branch and the excitatory/inhibitory connection branches. Each excitatory or inhibitory input will add a branch, with the variable conductance controlled by the parameters, step $\sigma$ and time constant $\tau$.

Event time is recorded when the membrane potential exceeds its threshold, $\theta$, and the potassium conductance is activated to induce the cell's refractory period. Thus, the computation is reduced by leaving out the details of action potential generation. The following equations describe the neuronmime model:

$$
\begin{gathered}
\tau_{\mathrm{m}} \frac{d V_{\mathrm{m}}}{d t}=-V_{\mathrm{m}}-g_{\mathrm{k}}\left(V_{\mathrm{m}}-E_{\mathrm{k}}\right) \\
-g_{\mathrm{ex}}\left(V_{\mathrm{m}}-E_{\mathrm{ex}}\right)-g_{\mathrm{in}}\left(V_{\mathrm{m}}-E_{\mathrm{in}}\right), \\
\tau_{\mathrm{k}} \frac{d g_{\mathrm{k}}}{d t}=-g_{\mathrm{k}}+b_{\mathrm{k}} S, \\
S=\left\{\begin{array}{l}
0, V_{\mathrm{m}}<0 \\
1, V_{\mathrm{m}} \geq 0
\end{array}\right\}
\end{gathered}
$$

where $g_{\mathrm{k}}=G_{\mathrm{k}} / G, g_{\mathrm{ex}}=G_{\mathrm{ex}} / G$, and $g_{\text {in }}=G_{\text {in }} / G ; G$, $G_{\mathrm{k}}, G_{\text {ex }}$, and $G_{\text {in }}$ represent respectively the conductances

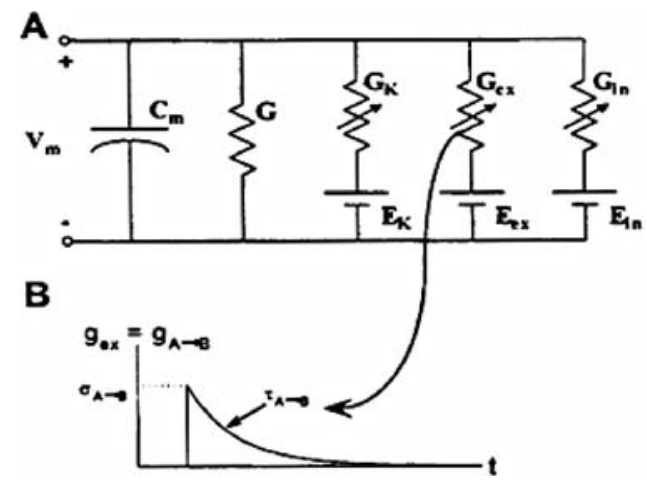

FIGURE 5. (A) $V_{m}$ is the membrane potential relative to rest; $C_{\mathrm{m}}$ is membrane capacitance; $G$ is resting conductance; $G_{\mathrm{k}}$ and $E_{\mathrm{k}}$ are variable conductance and reversal potential of potassium; $G_{\mathrm{ex}}$ /in and $E_{\mathrm{ex} / \mathrm{in}}$ are excitatory/inhibitory synaptic conductance and reversal potential. (B) Expression of connection synapse conductance. Target cell will undergo a step increase and exponential decay, time constant in response to input spikes.

of neuron membrane, potassium channel, excitatory synaptic inputs and inhibitory synaptic inputs. The latter three are normalized by $G$ to simplify the equations. $V_{\mathrm{m}}$ is the membrane potential. $E_{\mathrm{k}}, E_{\mathrm{ex}}$, and $E_{\mathrm{in}}$ represent the reversal potentials. $\tau_{\mathrm{m}}$ and $\tau_{\mathrm{k}}$ are the membrane time constant and the refractory time constant. Equation (1) states the current fluxes across the neuronal membrane. Equation (2) states the change of the potassium conductance, where $b_{\mathrm{k}}$ represents the sensitivity to potassium conductance. $S$ is the spiking variable with two alternative values: 1 indicates a cell is firing.

For target cell B, the variable conductance that represents synapses from source cells A is described by

$$
\tau_{\mathrm{A} \rightarrow \mathrm{B}} \frac{d g_{\mathrm{A} \rightarrow \mathrm{B}}}{d t}=-g_{\mathrm{A} \rightarrow \mathrm{B}}+\sigma_{\mathrm{A} \rightarrow \mathrm{B}} \sum_{i=1}^{N_{\mathrm{A} \rightarrow \mathrm{B}}} S_{\mathrm{A}_{i}}
$$

where $S_{\mathrm{A}}$ are the input spikes.

\section{Parameter Values}

In the model, we used four intrinsic $\left(\tau_{\mathrm{m}}, \theta, b_{\mathrm{k}}\right.$, and $\left.\tau_{\mathrm{k}}\right)$ and five connection parameters $\left(C_{\mathrm{A} \rightarrow \mathrm{B}}, \mathrm{BW} \mathrm{A}_{\mathrm{A} \rightarrow \mathrm{B}}, N_{\mathrm{A} \rightarrow \mathrm{B}}\right.$, $\sigma_{\mathrm{A} \rightarrow \mathrm{B}}$, and $\left.\tau_{\mathrm{A} \rightarrow \mathrm{B}}\right)$. The intrinsic parameter set describes the membrane properties of the cell, which determines the steady firing rate and the threshold sound pressure level of the single cell. The connection parameter set describes

TABLE 2. Intrinsic parameters.

\begin{tabular}{lrrrcccc}
\hline Population & $\tau_{\mathrm{m}}(\mathrm{ms})$ & $\theta(\mathrm{mV})$ & $b_{\mathrm{k}}$ & $\tau_{\mathrm{k}}(\mathrm{ms})$ & $E_{\mathrm{k}}(\mathrm{mV})$ & $E_{\mathrm{ex}}(\mathrm{mV})$ & $E_{\mathrm{in}}(\mathrm{mV})$ \\
\hline W-cells & 5.0 & 4.0 & 2.00 & 1.0 & -10 & +70 & -10 \\
I2-cells & 6.0 & 14.5 & 1.75 & 1.0 & -10 & +70 & -10 \\
P-cells & 10.0 & 7.5 & 2.00 & 1.0 & -10 & +70 & -10 \\
\hline
\end{tabular}


TABLE 3. Connection parameters.

\begin{tabular}{lccccc}
\hline $\begin{array}{c}\text { Connection } \\
\mathrm{A} \rightarrow \mathrm{B}\end{array}$ & $\begin{array}{c}\mathrm{C}_{\mathrm{A} \rightarrow \mathrm{B}} \\
\text { (octave) }\end{array}$ & $\begin{array}{c}\mathrm{BW}_{\mathrm{A} \rightarrow \mathrm{B}} \\
\text { (octave) }\end{array}$ & $\mathrm{N}_{\mathrm{A} \rightarrow \mathrm{B}}$ & $\sigma_{\mathrm{A} \rightarrow \mathrm{B}}$ & $\tau_{\mathrm{A} \rightarrow \mathrm{B}}(\mathrm{ms})$ \\
\hline $\mathrm{AN} \rightarrow \mathrm{W}$ & 0.0 & Varies & 140 & 0.06 & 10 \\
$\mathrm{AN} \rightarrow \mathrm{I} 2$ & 0.0 & 0.4 & 48 & 0.55 & 10 \\
$\mathrm{AN} \rightarrow \mathrm{P}$ & 0.0 & 0.4 & 48 & Varies & 10 \\
$\mathrm{~W} \rightarrow \mathrm{I} 2$ & 0.0 & 0.1 & 15 & 1.40 & 10 \\
$\mathrm{~W} \rightarrow \mathrm{P}$ & $0 / 0.2$ & 0.1 & 15 & Varies & 10 \\
$\mathrm{I} \rightarrow \mathrm{P}$ & $-0.2 / 0$ & 0.6 & 21 & 0.1 & 1 \\
$\mathrm{NSA} \rightarrow \mathrm{P}$ & $\mathrm{n} / \mathrm{a}$ & $\mathrm{n} / \mathrm{a}$ & 15 & 0.15 & 3 \\
\hline
\end{tabular}

the connections between two groups of cells; this is used to specify the connectivity within the neuronal circuitry of the model. We used the intrinsic values in the former work of Hancock and Voigt ${ }^{6}$ (Table 2), as they have little or no effect on the spectral response characteristics.
MacGregor ${ }^{11}$ pointed out that the steady state effect of one population on its target is proportional only to the product $N_{\mathrm{A} \rightarrow \mathrm{B}} \sigma_{\mathrm{A} \rightarrow \mathrm{B}} \tau_{\mathrm{A} \rightarrow \mathrm{B}}$, which enabled us to set only one parameter from these three to be a free parameter; here we chose $\sigma_{\mathrm{A} \rightarrow \mathrm{B}}$, the strength from cell A to B. Thus, only
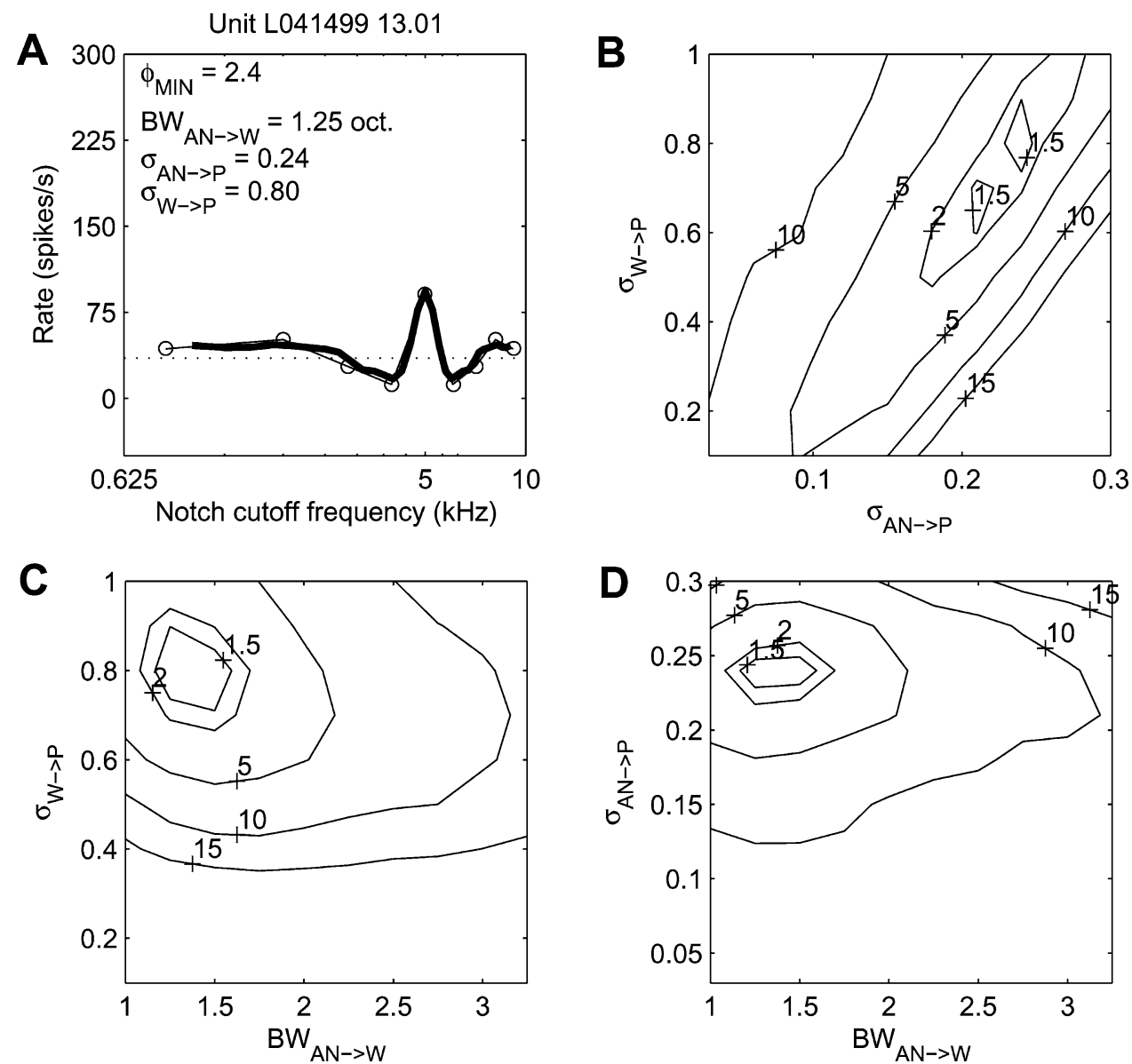

FIGURE 6. Best fit of Unit L041499 13.01 and parameter sensitivity analysis. (A) Best fit of the model to physiological data. The best frequency of physiological data and the center frequency of the model data have been lined up through simply translation of the physiological data rate vs. cutoff frequency plot. The circles represents the physiological data and the solid line represents the best fit of the model. The dashed line represents the average spontaneous rate of the physiological data. The minimum error $\Phi_{\min }$ and the values of the three important parameters of the best fit are shown in the upper left. (B)-(D) Contours of equal $\Phi / \Phi_{\min }(1.5$, $2,5,10$, and 15, from inside to outside) showing the sensitivity of the fit to the parameter values. BW $\mathrm{AN} \rightarrow \mathrm{W}, \sigma_{\mathrm{AN} \rightarrow \mathrm{P}}$, and $\sigma_{\mathrm{W} \rightarrow \mathrm{P}}$ are held to be the value in the best fit corresponsively in plots (B), (C), and (D). 

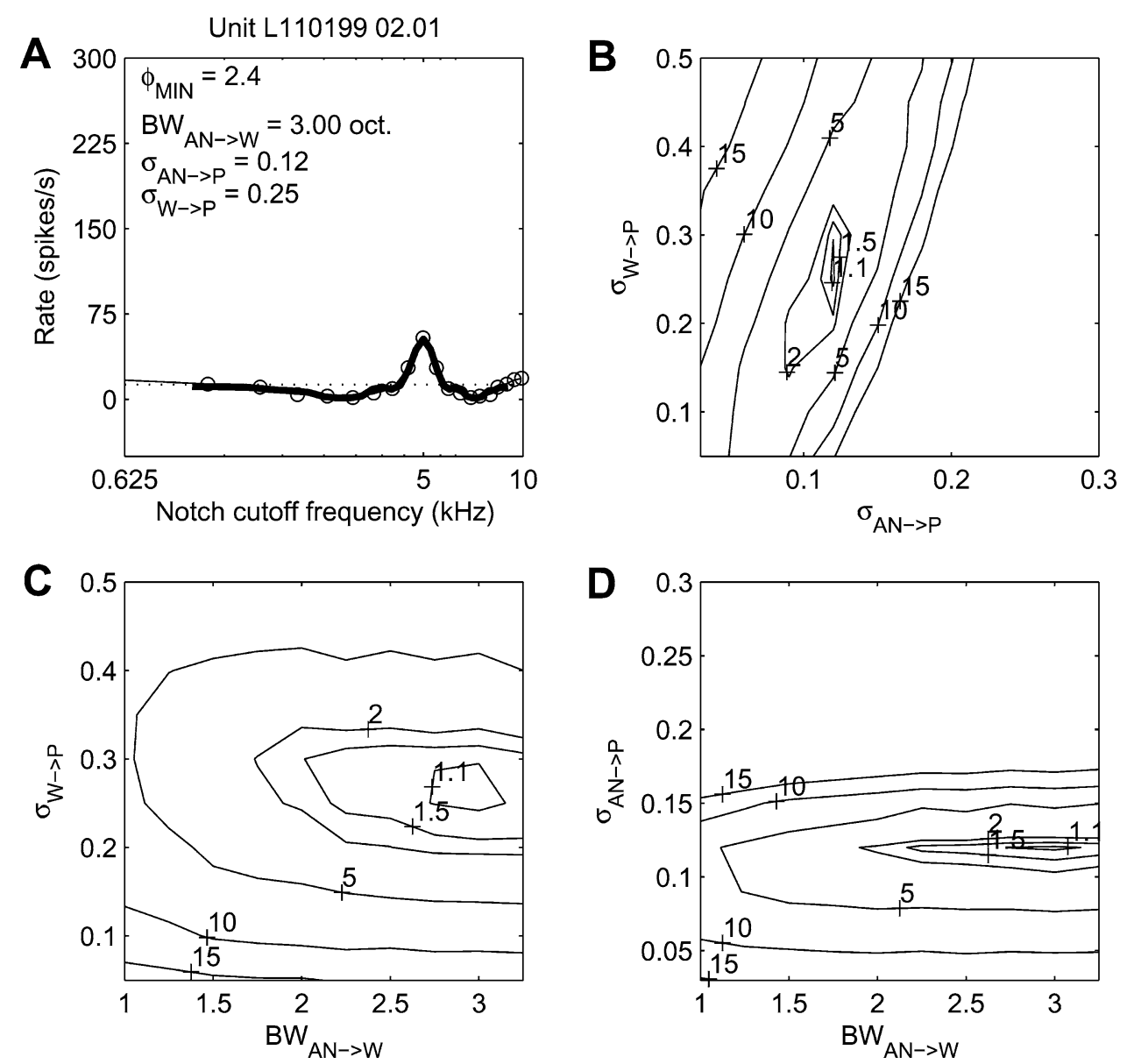

FIGURE 7. Best fit of Unit L110199 2.01 and parameter analysis. Organized as in Fig. 6 except that in the contours plots (B-D) equal $\Phi / \Phi_{\min }$ of 1.1 was added to show the center.

three connection parameters, $\sigma_{\mathrm{A} \rightarrow \mathrm{B}}, \mathrm{BW}_{\mathrm{A} \rightarrow \mathrm{B}}$, and $C_{\mathrm{A} \rightarrow \mathrm{B}}$ were taken into account in the simulations. The values of the parameters are listed in Table 3.

As in Table 3, three parameters were set to be main parameters that vary: $\mathrm{BW}_{\mathrm{AN} \rightarrow \mathrm{W}}, \sigma_{\mathrm{AN} \rightarrow \mathrm{P}}$, and $\sigma_{\mathrm{W} \rightarrow \mathrm{P}}$. Center offset $C_{\mathrm{I} 2 \rightarrow \mathrm{P}}$ and $C_{\mathrm{W} \rightarrow \mathrm{P}}$ were set to two possible values $0 /-0.2$ octaves and $0 / 0.2$ octaves respectively according to the physiological data in cross-correlation studies. $\sigma_{\mathrm{I} 2 \rightarrow \mathrm{P}}$ was set to 0.1 to obtain type III RM properties. Non-specific afferent firing rate was set to 80,90 , or 120 spikes/s according to the various spontaneous rates of type III units.

\section{Auditory Nerve Model}

AN-fibers were based on the model described by Carney. ${ }^{1}$ The input to the AN-fiber is a sound pressure signal and the output is a spike train. Each fiber consists of a gammatone filter that provides the frequency selectivity, a nonlinear process that generates an inner hair cell potential and a compartmental model of neurotransmitter release. This in turn creates an instantaneous firing probability. AN-fiber thresholds and spontaneous activity rates were randomly assigned from physiological distributions of these parameters. ${ }^{5}$

In order to save time, all stimuli were processed by the auditory nerve filter bank once and the spike time responses were saved for use in subsequent simulations of the DCN circuitry.

\section{Simulation Protocol and Data Analysis}

All simulations were carried out on an IBM pSeries 655 , which is a 48-processor system composed of six nodes. Each p655 node consists of eight Power4 processors running at $1.1 \mathrm{GHz}$ and sharing $16 \mathrm{~GB}$ of memory. There are three levels of cache on this machine. Each processor has a $32 \mathrm{~KB} \mathrm{L1}$ cache and then each pair of processors share a $1.41 \mathrm{MB}$ L2 cache, and each p655 node shares a 128 MB L3 cache.

To create RMs, the model was stimulated by $50-\mathrm{ms}$ tone bursts presented every $250 \mathrm{~ms}$ with a 32 -ms delay while sound pressure levels varied from 0 to $60 \mathrm{~dB}$ SPL in $2 \mathrm{~dB}$ SPL steps and the frequency varied in 0.1 octave steps within a three octave band above and below $5 \mathrm{kHz}$. Thus, 

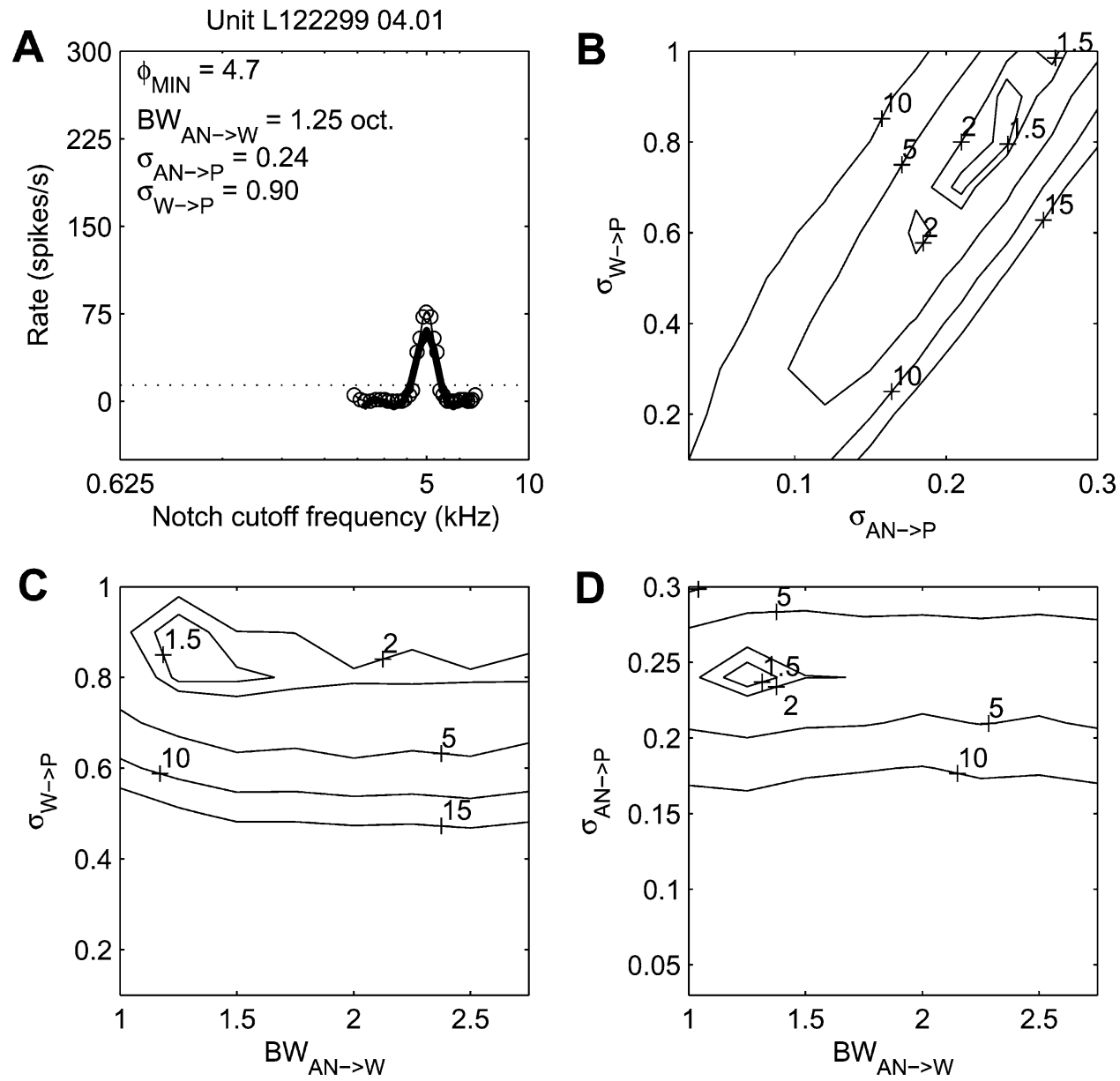

FIGURE 8. Best fit of Unit L122299 4.01 and parameter analysis. Organized as in Fig. 6.

there are 31 frequency slices in one RM simulation. The spikes of the last $40 \mathrm{~ms}$ of each trial were used to compute the spontaneous rate and the spikes of the last $40 \mathrm{~ms}$ of each tone burst were used to compute the driven rate. In this paper, RMs composed of rate vs. frequency curves for seven levels were plotted (0-60 dB SPL in $10 \mathrm{~dB}$ SPL steps). This is because the physiological data were taken only to $60 \mathrm{~dB}$ SPL due to increasing recording artifacts above these levels. At each level, the horizontal line represents the unit's spontaneous discharge rate; excitation and inhibition are shown as rates above and below this line, respectively. The excitatory region was filled black and the inhibitory region left blank.

We used the same notch noises and broadband noise stimuli to obtain rate-level data as in Hancock and Voigt. ${ }^{6}$ In the simulation the sound level was varied from 0 to $90 \mathrm{~dB}$ SPL in $2 \mathrm{~dB}$ SPL steps and the noise bursts were presented for $200 \mathrm{~ms}$ in 1000-ms trials.

To obtain rate vs. notch cutoff frequency plots, the firing rates from 46 to $54 \mathrm{~dB}$ SPL were averaged to capture the most inhibitory area, consistent with Hancock and Voigt. ${ }^{6}$ These plots are sometimes referred to as "Mexican Hats."

\section{RESULTS}

\section{Fit of the Model to Physiological Data and Parameter} Sensitivity Analysis

Discharge rate versus cutoff frequency plots were used to evaluate the effectiveness of our DCN model quantitatively when fit to the physiological data obtained by Parsons et al. ${ }^{15}$ The physiological discharge rate versus cutoff frequency plots were translated along the frequency axis to align the $\mathrm{BF}$ of the unit to that of the model at $5 \mathrm{kHz}$ without shape change in logarithmic frequency plots. This operation was done mathematically by scaling the cutoff frequencies by $\mathrm{CF} / \mathrm{BF}$, where $\mathrm{CF}$ is the center frequency of model and BF is the best frequency of physiological data. This operation also made the after-translation physiological data look like that obtained by notch noise stimuli centered at $5 \mathrm{kHz}$ while notch widths scaled by the factor $\mathrm{CF} / \mathrm{BF}$. Then linear interpolation was used to obtain "new" physiological data corresponding to model data cutoff frequencies and this made comparison of fits among different units possible. Here we used an objective function, $\Phi$, to demonstrate 
TABLE 4. Data fits for all 15 type III units.

\begin{tabular}{|c|c|c|c|c|c|c|c|c|c|c|c|}
\hline & In Ref & Unit & $\mathrm{BF}$ & SR & $\Phi_{\min }$ & NSA & $\mathrm{C}_{\mathrm{W} \rightarrow \mathrm{P}}$ & $\mathrm{C}_{\mathrm{I} 2 \rightarrow \mathrm{P}}$ & $\mathrm{BW}_{\mathrm{AN} \rightarrow \mathrm{W}}$ & $\sigma_{\mathrm{AN} \rightarrow \mathrm{P}}$ & $\sigma_{\mathrm{W} \rightarrow \mathrm{P}}$ \\
\hline 1 & Fig. $3 A$ & $\begin{array}{c}\text { L122299 } \\
3.01\end{array}$ & 4.3 & 38 & 3.4 & 90 & 0 & 0 & $3.25^{\mathrm{a}}$ & 0.25 & 0.90 \\
\hline 2 & Fig. 3B & $\begin{array}{c}\text { L021199 } \\
1.01\end{array}$ & 4.7 & 77 & 3.8 & 120 & 0.2 & 0 & $3.25^{a}$ & 0.45 & 1.80 \\
\hline 3 & Fig. 3C & $\begin{array}{c}\text { L110199 } \\
2.01\end{array}$ & 2.6 & 13 & 2.4 & 80 & 0 & 0 & 3.00 & 0.12 & 0.25 \\
\hline 4 & Fig. 4A & $\begin{array}{c}\text { L111799 } \\
9.01\end{array}$ & 4.9 & 9 & 2.8 & 80 & 0.2 & -0.2 & 2.00 & 0.12 & 0.25 \\
\hline 5 & Fig. 4B & $\begin{array}{c}\text { L122299 } \\
4.01\end{array}$ & 11.0 & 14 & 4.7 & 80 & 0 & -0.2 & 1.25 & 0.24 & 0.90 \\
\hline 6 & Fig. 4C & $\begin{array}{r}\text { L110199 } \\
11.02\end{array}$ & 4.2 & 12 & 11.0 & 80 & 0 & 0 & $1.00^{\mathrm{a}}$ & 0.15 & $0.05^{a}$ \\
\hline 7 & Fig. 4D & $\begin{array}{c}\text { L102599 } \\
7.01\end{array}$ & 10.2 & 26 & 5.1 & 90 & 0 & 0 & 1.50 & 0.25 & 0.70 \\
\hline 8 & Fig. 4E & $\begin{array}{c}\text { L041499 } \\
16.01\end{array}$ & 3.8 & 30 & 8.1 & 80 & 0.2 & -0.2 & 2.50 & 0.12 & 0.20 \\
\hline 9 & Fig. $4 \mathrm{~F}$ & $\begin{array}{c}\text { L021199 } \\
4.01\end{array}$ & 7.1 & 15 & 2.8 & 80 & 0 & 0 & $3.25^{a}$ & 0.18 & 0.25 \\
\hline 10 & Fig. 4G & $\begin{array}{c}\text { L102599 } \\
8.01\end{array}$ & 6.4 & 22 & 5.6 & 80 & 0 & 0 & 1.50 & 0.12 & 0.40 \\
\hline 11 & Fig. $4 \mathrm{H}$ & $\begin{array}{c}\text { L041499 } \\
13.01\end{array}$ & 1.2 & 35 & 2.4 & 80 & 0 & 0 & 1.25 & 0.24 & 0.80 \\
\hline 12 & Fig. 4 I & $\begin{array}{c}\text { L041499 } \\
1.02\end{array}$ & 1.3 & 9 & 9.8 & 80 & 0.2 & 0 & $3.25^{a}$ & 0.06 & 0.15 \\
\hline 13 & Fig. 4J & $\begin{array}{c}\text { L041499 } \\
1.01\end{array}$ & 1.2 & 49 & 5.8 & 80 & 0 & -0.2 & $1.00^{\mathrm{a}}$ & 0.24 & 0.90 \\
\hline 14 & Fig. $4 \mathrm{~K}$ & $\begin{array}{c}\text { L040700 } \\
3.01\end{array}$ & 11.4 & 20 & 1.3 & 80 & 0.2 & 0 & 3.00 & 0.24 & 0.25 \\
\hline 15 & Fig. 4L & $\begin{array}{c}\text { L040700 } \\
7.01\end{array}$ & 15.1 & 24 & 0.4 & 120 & 0 & 0 & 2.75 & 0.45 & $2.00^{\mathrm{a}}$ \\
\hline
\end{tabular}

Note. Ref. refers to Parsons et al. ${ }^{15}$; BF in $\mathrm{kHz}, \mathrm{SR}$ in spikes/s, NSA in spikes/s.

aThe parameter value reached the parameter limit.

quantitatively the fit of one unit, or for comparisons across the units as done by Hancock and Voigt ${ }^{6}$ :

$$
\Phi=\frac{1}{N} \sum_{i=1}^{N}\left(\frac{y_{\mathrm{d}}(i)-y_{\mathrm{m}}(i)}{\max \left(y_{\mathrm{d}}\right)}\right)^{2} \times 500,
$$

where $y_{\mathrm{d}}$ and $y_{\mathrm{m}}$ are the mean firing rates in the physiological data and model results respectively, and $N$ is the total number of cutoff frequencies used in the comparison. The value of the objective function is scaled by 500 to make it more readable, consistent with Hancock and Voigt. ${ }^{6}$

The three parameters $\mathrm{BW}_{\mathrm{AN} \rightarrow \mathrm{W}}, \sigma_{\mathrm{AN} \rightarrow \mathrm{P}}$, and $\sigma_{\mathrm{W} \rightarrow \mathrm{P}}$, were systematically varied to get the "best fit" (minimum $\left.\Phi, \Phi_{\min }\right)$ and also to show contours of equal goodness or quality of fit. Center offsets $C_{\mathrm{I} 2 \rightarrow \mathrm{P}}$ and $C_{\mathrm{W} \rightarrow \mathrm{P}}$, were set to 0 or -0.2 octaves and 0 or 0.2 octaves respectively, and the fitting results were only slightly different with similar $\Phi_{\min }$ when best fits were obtained. Non-specific afferent (NSA) firing rate was set to 80,90 , or 120 spikes/s to adjust to the various spontaneous rates of type III units and this improved the fits, especially for some low-spontaneous rate units. An alternative way to adjust spontaneous rate was to vary the connection strength of NSA to P-cells as in Hancock and Voigt. ${ }^{6}$ These two methods show no difference from each other since P-cells are the only targets of the NSA and the different firing rates of NSA or the strength of the connection between NSA and P-cells will only affect the total NSA excitatory inputs to P-cells.

Figures 6-8A show the best fits to three physiological units labeled Unit L041499 13.01, Unit L110199 2.01 and Unit L122299 4.01. Figures 6-8B-D are contours showing parameter sensitivity analyses of these parameters. As shown in the figures, $\mathrm{BW}_{\mathrm{AN} \rightarrow \mathrm{W}}$ varied from 1.0 to 3.25 octaves in steps of 0.25 octaves; $\sigma_{\mathrm{AN} \rightarrow \mathrm{P}}$ varied from 0.05 to 0.5 or from 0.03 to 0.3 ; and $\sigma_{\mathrm{W} \rightarrow \mathrm{P}}$ varied from 0.1 to 1.0 or from 0.05 to 0.5

Very good fits are obtained for these three units. Not only are the values of $\Phi_{\min }$ small, but the centers of the contours are shown clearly and the contours themselves resemble ellipses.

Table 4 shows the results for all 15 units. Of the 15 units from Parsons et al., ${ }^{15}$ eight units had best fits within closed ellipses. Another six units also gave good fits, although the $\mathrm{BW}_{\mathrm{AN} \rightarrow \mathrm{W}}$ parameter in these cases was actually at its limit 
A

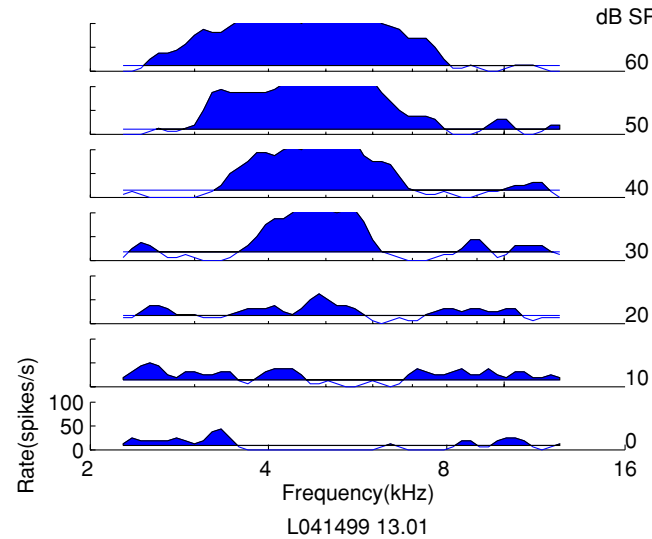

C

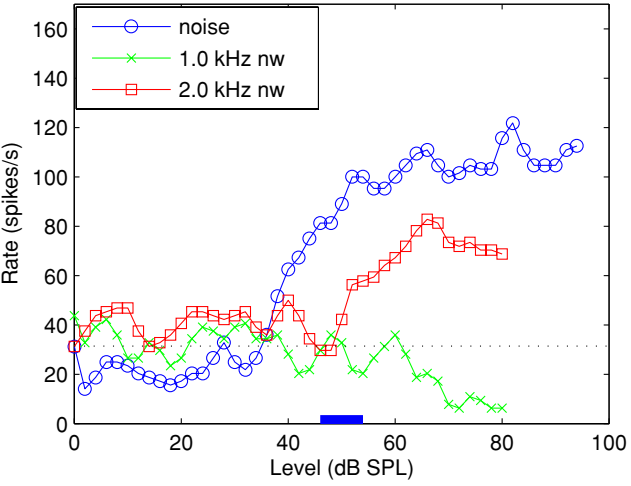

B

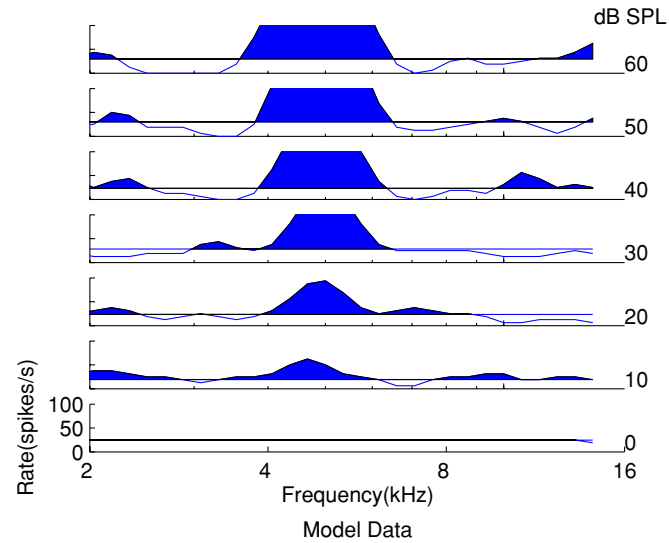

D

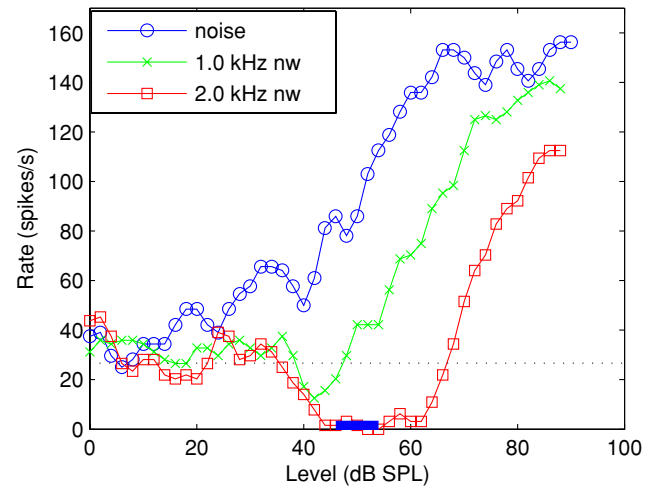

FIGURE 9. Response maps and rate-level plots for notch noise stimuli. (A, B) Response map composed of rate vs. frequency curves plot of Unit L041499 13.01 and the model using the set of best fit parameters in Fig. 6 . The best frequency of this unit is $1.2 \mathrm{kHz}$ and has been moved to $5 \mathrm{kHz}$ with the same method in "Mexican Hat" plots for easier comparison to model RM. (C, D) Rate-level curves of broad-band noise and notch noise responses of the unit and the model data.

(four reaching the upper limit and one reaching the lower limit of the parameter range, one reaching the upper limit of $\left.\sigma_{\mathrm{W} \rightarrow \mathrm{P}}\right)$. These 14 units had values of $\Phi_{\min }<9.8$, which shows that the model is robust. The final unit was difficult to fit because the rate vs. notch noise cutoff frequency plot showed additional features (see Parsons et al., Fig. 4C). ${ }^{15}$

\section{Comparisons Between Physiological Data and Model} Data: Response Maps and Notch Noise Rate-Level Curves

Since the rate vs. notch noise cutoff frequency curves are the functions that were modeled, to what extent do the model RMs and notch noise rate-level curves resemble their physiological counterparts? Figures 9-11A and B show the physiological RMs together with the model RMs for the units of Figures 6-8. The model RMs were simulated using the model parameters set found when $\Phi=\Phi_{\min }$. For two sets of RMs we found that the model RMs show insufficient excitatory regions across the frequency axis when highlevel tone bursts are given. This occurs in another five units where the physiological data show wide excitatory regions and indicates that our model parameters may need adjustment to get better tone responses for these units. All three model RMs, however, show nice center excitatory regions and sideband inhibitory regions, which is characteristic of type III units.

Figures 9-11C and D show the rate-level curves corresponding to the BBN and NN stimuli. For Unit L041499 13.01 and Unit L110199 2.01 (Figures 9 and 10), the maximum notch widths could not reach $8.0 \mathrm{kHz}$ since the units had low BFs (1.2 and $2.6 \mathrm{kHz}$ ). Unit L122299 4.01 has a higher $\mathrm{BF}$ at $11.0 \mathrm{kHz}$ and the notch width was varied from 0.5 to $8.5 \mathrm{kHz}$ as in Parsons et al..$^{15}$ In general, the responses to BBN are greatest in both model and physiological data, but there are great differences in shape, maximum rate, saturation rates and thresholds. The model's curves for the NN stimuli are qualitatively similar to the physiology, but again several differences are observed. The model seems to be providing more inhibition than that found in the physiology.

\section{DISCUSSION}

Spirou and Young ${ }^{18}$ showed that type IV units in the cat DCN (projection neurons) are notch detectors. Hancock and Voigt $^{6}$ used a computational model of cat DCN circuitry, whose P-cells had type IV response properties to show that these also have notch noise sensitivity and that the 

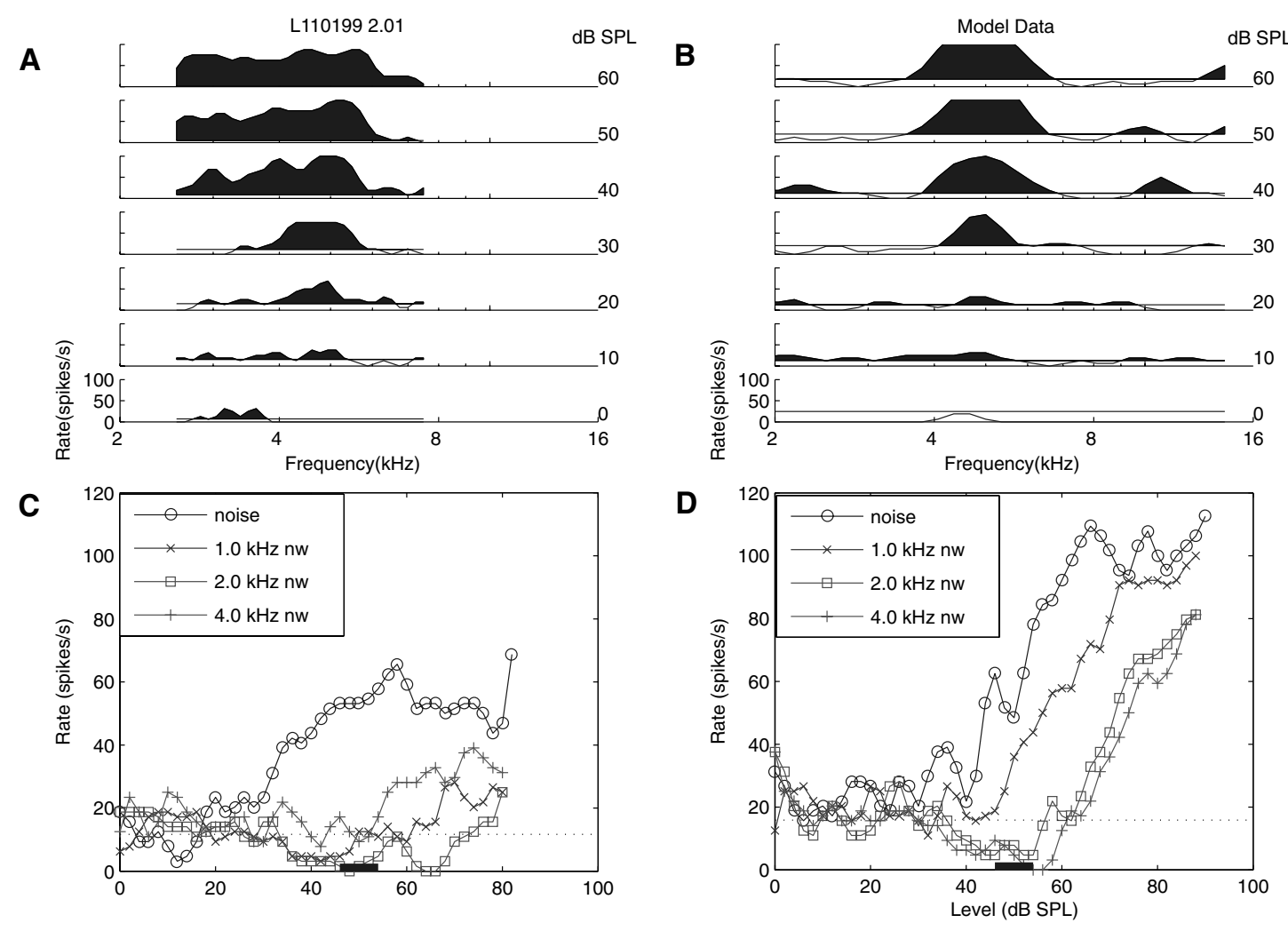

FIGURE 10. Response maps and rate-level plots responding to notch noise stimuli. Unit L110199 2.01. The best frequency of this unit is 2.6 kHz. Picture is organized as in Fig. 9.

variability seen in the units' responses to notch noise are accounted for by parameter variations rather than different circuit arrangements. The gerbil DCN shows far fewer type IV units than the cat and the projection neurons in gerbil DCN are predominately type III units. Parsons et al. ${ }^{15}$ showed that gerbil type III units are sensitive to notch noises in much the same way as cat DCN type IV units. Thus, it appears that $\mathrm{DCN}$ projection neurons from both species are doing the same analysis on notch noise and thus may play similar roles in sound source detection in the median plane.

This study demonstrates that the same computational model used to model the notch noise behavior in cat type IV units can be used to model type III unit notch noise sensitivity in gerbil DCN. The fact that we can use the same neural circuit to model the DCN in both species suggests that perhaps this aspect of the DCN neural circuit is invariant across species.

The Parsons et al. ${ }^{15}$ data set of 15 type III units provided a rich database to test our model. In all, the model provided reasonable fits to 14 type III units. Prior to this, notch noise responses from only three type IV units from cat were available for modeling. ${ }^{6,18}$ The objective function used here is the same one used in Hancock and Voigt ${ }^{6}$; this allowed direct comparisons to that study. In this study, the RMs of the model P-cells with the best-fit parameters were compared to the physiological units' RMs. The model RMs had excitatory center regions with inhibitory surrounds. These are similar to the physiological RMs and correspond to type III units. The model rate-level curves to broadband noise were similar to the physiological data, but the ratelevel curves to notch noise showed many differences. Of course, these data are not part of the objective function used and so we would expect differences. It is possible to modify the objective function to include the rate-level curves, the RMs, or both, and this may be done in the future.

Our model also shows reduced sensitivity to $\mathrm{BW}_{\mathrm{AN} \rightarrow \mathrm{W}}$ in half of the units. Four units reached the upper limit of $\mathrm{BW}_{\mathrm{AN} \rightarrow \mathrm{W}}=3.25$ octave and two units reached the lower limit of $\mathrm{BW}_{\mathrm{AN} \rightarrow \mathrm{W}}=1.00$ octave, which indicates that better fits might be obtained by extending the range of $\mathrm{BW}_{\mathrm{AN} \rightarrow \mathrm{W}}$. This is not possible in the present version of the model, which is limited to a four octave frequency range.

Non-specific afferent (NSA) firing rate affected the $\Phi_{\min }$ in a significant way for some units. This was not true, however, for most of the units, where NSA firing rates of 80, 90, and 120 spikes/s produced similar $\Phi_{\min }$. The best-fit parameter values and sensitivity plots, however, would change significantly when different rates applied. In general, the parameters of the best fits showed no specific relations to unit $\mathrm{BF}$ or spontaneous rates. 

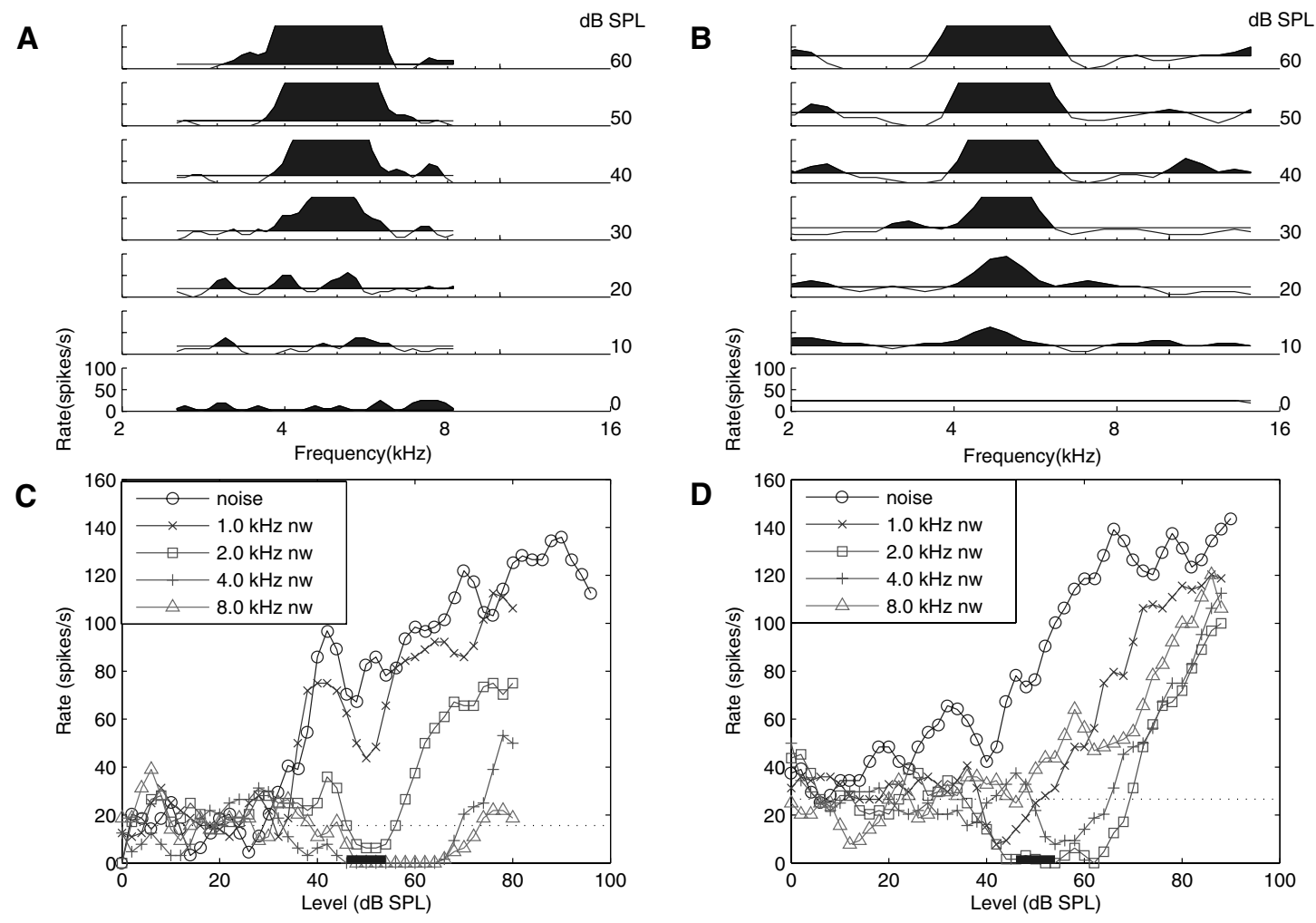

FIGURE 11. Response maps and rate-level plots responding to notch noise stimuli. Unit L122299 4.01. The best frequency of this unit is $11.0 \mathrm{kHz}$. Picture is organized as in Fig. 9.

The DCN computational models for cat and gerbil are identical in form and differ only in connectivity. The model is still incomplete, however, in that it lacks model cartwheel cells, granule cells and other cell types known to exist. The $\mathrm{CN}$ also receives input from somatosensory neurons that subserve tactile and kinesthetic sensations from the trigeminal ganglion ${ }^{16}$ the interpolar and caudal spinal trigeminal ganglion, ${ }^{8,25}$ cuneate nucleus ${ }^{19}$ as well as from other locations. These indicate that in addition to auditory function, the $\mathrm{CN}$ is involved in sensory integration at a very early stage in the brain. These are not currently in the model, but provide rich directions to explore in the future.

\section{ACKNOWLEDGMENTS}

This work was supported by grant from the NIDCD and Boston University's Biomedical Engineering department and Hearing Research Center. We appreciate the comments on earlier versions of this manuscript from Dr. Barbara Shinn-Cunningham, Rapeechai Navawongse, and Seth Newburg.

\section{REFERENCES}

${ }^{1}$ Carney, L. H. A model for the responses of low-frequency auditory-nerve fibers in cat. J Acoust. Soc. Am. 93:401-417, 1993.
${ }^{2}$ Davis, K. A., J. Ding, T. E. Benson, and H. F. Voigt. Response properties of units in the dorsal cochlear nucleus of unanesthetized decerebrate gerbil. J. Neurophysiol. 75:14111431, 1996.

${ }^{3}$ Ding, J., T. E. Benson, and H. F. Voigt. Acoustic and currentpulse responses of identified neurons in the dorsal cochlear nucleus of unanesthetized, decerebrate gerbils. J. Neurophysiol. 82:3434-3457, 1999.

${ }^{4}$ Evans, E. F., and P. G. Nelson. The responses of single neurones in the cochlear nucleus of the cat as a function of their location and the anaesthetic state. Exp. Brain Res. 17:402-427, 1973.

${ }^{5}$ Hancock, K. E., K. A. Davis, and H. F. Voigt. Modeling inhibition of type II units in the dorsal cochlear nucleus. Biol. Cybern. 76:419-428, 1997.

${ }^{6}$ Hancock, K. E., and H. F. Voigt. Wideband inhibition of dorsal cochlear nucleus type IV units in cat: A computational model. Ann. Biomed. Eng. 27:73-87, 1999.

${ }^{7}$ Hancock, K. E., and H. F. Voigt. Intracellularly labeled fusiform cells in dorsal cochlear nucleus of the gerbil. I. Physiological response properties. J. Neurophysiol. 87:2505-2519, 2002.

${ }^{8}$ Itoh, K., H. Kamiya, A. Mitani, Y. Yasui, M. Takada, and N. Mizuno. Direct projections from the dorsal column nuclei and the spinal trigeminal nuclei to the cochlear nuclei in the cat. Brain Res. 400:145-150, 1987.

${ }^{9}$ Koerber, K. C., R. R. Pfeiffer, W. B. Warr, and N. Y. Kiang. Spontaneous spike discharges from single units in the cochlear nucleus after destruction of the cochlea. Exp. Neurol. 16:119130, 1966.

${ }^{10}$ MacGregor, R. J. Neural and Brain Modeling. San Diego, CA: Academic, 1987. 
1946

X. ZHENG AND H. F. VOIGT

${ }^{11}$ MacGregor, R. J. Theoretical Mechanics of Biological Neural Networks. San Diego, CA: Academic, 1993.

${ }^{12}$ Maki, K., S. Furukawa, and T. Hirahara. Acoustical cues for sound localization by gerbils in an ecologically realistic environment. Assoc. Res. Otolaryngol. Abs. 26:352, 2003.

${ }^{13}$ Musivant, A. D., and R. A. Butler. Influence of monaural spectral cues on binaural localization. J. Acoust. Soc. Am. 77:202-208, 1985.

${ }^{14}$ Nelken, I., and E. D. Young. Two separate inhibitory mechanisms shape the responses of dorsal cochlear nucleus type IV units to narrowband and wideband stimuli. J. Neurophysiol. 71:2446-2462, 1994.

${ }^{15}$ Parsons, J. E., E. Lime, and H. F. Voigt. Type III units in the gerbil dorsal cochlear nucleus may be spectral notch detectors. Ann. Boomed. Eng. 29:887-896, 2001.

${ }^{16}$ Shore, S. E., Z. Vars, N. L. Wys, and R. A. Altschuler. Trigeminal ganglion innervates the auditory brainstem. J. Comp. Neural. 419:271-285, 2000.

${ }^{17}$ Shofner, W. P., and E. D. Young. Excitatory/inhibitory response types in the cochlear nucleus: relationships to discharge patterns and responses to electrical stimulation of the auditory nerve. $J$. Neurophysiol. 54:917-939, 1985.

${ }^{18}$ Spirou, G. A., and E. D. Young. Organization of dorsal cochlear nucleus type IV unit response maps and their relationship to activation by bandlimited noise.J.Neurophysiol. 66:1750-1768, 1991.

${ }^{19}$ Weinberg, R. J., and A. Rustioni. A cuneocochlear pathway in the rat. Neuroscience 20:209-219, 1987.

${ }^{20}$ Winter, I. M., and A. R. Palmer. Level dependence of cochlear nucleus onset unit responses and facilitation by secon tones or broadband noise. J. Neurophysiol. 73:141-159, 1995.

${ }^{21}$ Young, E. D. Identification of response properties of ascending axons from dorsal cochlear nucleus. Brain Res. 200:23-37, 1980.

${ }^{22}$ Young, E. D., and W. E. Brownell. Responses to tones and noise of single cells in dorsal cochlear nucleus of unanesthetized cats. J. Neurophysiol. 39:282-300, 1976.

${ }^{23}$ Young, E. D., and H. F. Voigt. Response properties of type II and type III units in the dorsal cochlear nucleus. Hear. Res. 6:153-169, 1982.

${ }^{24}$ Zheng, X., and H. F. Voigt. A modeling study of notch noise responses of type III units in the gerbil dorsal cochlear nucleus. Assoc. Res. Otolaryngol. Abs. 440, 2005.

${ }^{25}$ Chou, J., and S. Shore. Projections from the trigeminal nuclear complex to the cochlear nuclei: A retrograde and anterograde tracing study in the guinea pig. J. Neurosci Res. 78:901-907, 2004. 